\title{
An Architecture for the Development of Function Block Oriented Engineering Support Systems
}

\author{
Kleanthis Thramboulidis and Chris Tranoris
}

\begin{abstract}
The always growing need, for innovative products, forces manufacturing plants to improve their ability to quickly respond to market requirements by designing competitive products and modifying existing ones. The function block concept, promises to improve productivity in terms of reuse, reliability, flexibility and interoperability. IEC, with standards like 61499, defines the basic concepts and a methodology for the design of modular, re-usable distributed Industrial Process, Measurement and Control Systems (IPMCSs). To support the whole life cycle of these systems, new function block oriented Engineering Support Systems (ESSs), are highly required. In this paper, we consider, the process of interconnecting heterogeneous fieldbus islands and, the design and implementation of integrated distributed applications over them. The architecture of the interworking unit was defined, to obtain interoperability in fieldbus level and to allow a uniform development of applications from the ESS point of view. A 4-layer architecture was also defined to facilitate the design and development of the new generation function block oriented ESSs.
\end{abstract}

\section{Introduction}

Today's rapidly changing market requirements impose the need of improving the agility of manufacturing systems. The always growing need for innovative products, forces manufacturing plants to improve their ability to quickly respond to market demands by designing competitive products and modifying existing ones. Until recently, most of the industrial control systems have been based either on traditional distributed control systems or on programmable logic controllers. In both cases, the systems are composed of monolithic applications that are almost impossible to integrate and even to expand. Modularity, flexibility, extensibility, reusability and interoperability are dimension slightingly addressed by many traditional proprietary engineering tools and products. Even more, the most of the traditional products and tools are far away from the new challenging technologies

Electrical \& Computer Engineering Dept. University of Patras, 26500

Patras, Greece, Tel: +30-61-997325; Fax: +30-61-997316

E-mail: \{thrambo, tranoris\} @ee.upatras.gr of Software Engineering. The IPMCS software industry increasingly faces today, the challenge of creating complex custom-made distributed control systems within time and budget, while high competition forces prices down.

Evolving standards, like IEC-61499 and the more recent IEC61804, define the basic concepts and a methodology for the design of modular, re-usable, distributed industrial process, measurement and control systems [1][2]. They define, the function block construct as the main building block of IPMCS applications, in a format that is independent of implementation. They also define, the way that function blocks can be used to define robust, re-usable software components that constitute the distributed IPMCSs. A function block may be primitive i.e. small enough to provide a solution to a small problem, such as the control of a valve, or composite, that is an aggregation of function blocks, i.e. big enough to control a major unit of plant. The function block construct is an abstraction mechanism that allows industrial algorithms to be encapsulated in a form that can be readily understood and applied by industrial engineers who are not specialists in the implementation of complex algorithms.

The above standards define also a methodology to be used by system designers to construct distributed control systems. It allows systems to be defined in terms of logically connected function blocks that run on different processing resources. Complete applications, can be built from networks of function blocks, formed by interconnecting their inputs and outputs. New generation function block oriented Engineering Support Systems (ESS), are highly required to support the whole life cycle of IPMCS applications. An ESS must support the engineer, in both the analysis and design phase as well as in the implementation phase. Using such a system, the engineer must be able to start with the analysis of the plant diagram so as to capture the control requirements. Then, he should be able to define the major areas of functionality and their interaction with the plant. During this task, he should be able to exploit function blocks provided by intelligent field devices, such as smart valves, but also to assign functionality into physical resources such as PLCs, instruments and controllers. All the above should be accomplished independent of the underlying communication subsystem and in the extreme case, where it is an aggregation of interconnect independent fieldbus segments, even from different vendors. 
Industry's solution to this problem is not very clear. For example, Softing's Profigate is an Ethernet-Profibus Gateway which consists of a PROFIBUS connector, a CPU board and an Ethernet TCP/IP software interface [3]. Another example is Siemens' SIMATIC OPC SERVER, which offers libraries for distant connectivity [4]. Two major reasons, though, put these solutions in question: first, the real-time transmission between two fieldbuses cannot be guaranteed, due to the Ethernet backbone, and second, these gateways offer only PROFIBUS connections.

Another commercial product that promises connectivity between independent fieldbus segments, is SST-X-Link from SST Network gateways [5]. Although this product seems promising, a great effort for configuration from user's part is required. It doesn't also offer the capability for remote configuring, diagnostics, monitoring, etc.

In the context of this work, we consider the process of interconnecting heterogeneous fieldbus islands and the need to design and implement, distributed function block oriented applications over them. The architecture of an interworking unit is defined so as to obtain interoperability in fieldbus level and allow a uniform development of applications independent of the underlying communication subsystems. This architecture must enable the interworking unit to:

- Meet the real-time constraints imposed by the fieldbus-tofieldbus communication channel

- Provide a uniform, independent of the underlying communication subsystems, interface to the ESS

- Provide a uniform interface to SCADA client systems

- Provide the interoperability mechanisms required by any communication channel

Our intention is to design an interoperable IEC61499compliant interworking unit that will provide the ability to utilize heterogeneous software environments, with IEC 61499-compliant software tools available from a wide selection of vendors. To capture the key abstractions, that will become the basic constructs for the development of the function block oriented ESS, we resulted in a 4-layer architecture that must be supported by the ESS. This architecture defines the main directions the ESS must address and should play an important role during its development.

The rest of this paper is structured as follows. In section 2 of this paper, we briefly refer to the function block approach. We introduce the function block construct as the primary building block for the design of IPMCS applications. Distributed applications are considered and a network topology is proposed to address the interoperability problem in fieldbus level. We next, in section 3, present the architecture of the interworking unit. The proposed architecture is described in terms of active modules, interaction mechanism and rules of interaction. Section 4 presents the 4-layer architecture that forms the basis for the development of our function block oriented ESS. Finally, in the last section, we conclude the paper.

\section{The Function Block approach}

\subsection{Function Block}

The latest trends in the development of IPMCSs are concentrated in the development of the evolving international standards IEC 61499 and IEC 61804. They both ameliorate the function block construct first introduced by the IEC 1131 standard on programming languages for programmable logic controllers, extending it to the new requirements of distributed control systems. The function block, as is defined by the IEC 61499 , is a functional unit of software, comprising an individual, named copy of the data structure specified by the function block type, which persists from one invocation of the function block to the next. The functionality of the function block is provided by means of algorithms, which process inputs and internal data and generate output data. A function block consists of a head and a body, as is shown in figure 1, where its graphical representation is given. The function block's head is connected to the event flows and its body to the data flows.

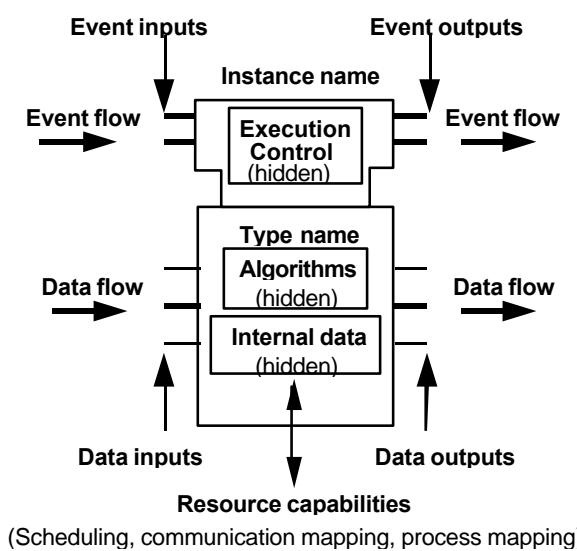

Figure 1. Graphical representation of the function block concept.

The function block concept, as is very well pointed out in [6], shares many of the well defined and already widely acknowledged benefits of the object concepts introduced by the Object Technology. Objects are stable; they reduce the complexity of the system and are highly reusable. The function block shares these attributes and constitutes a well-established concept for defining robust, re-usable IPMCS software components. The function block construct is an abstraction mechanism that allows industrial algorithms to be encapsulated in a form that can be readily understood and applied by industrial engineers, who are not specialists in the implementation of complex algorithms. 


\subsection{IPMCS applications}

The IEC 61499 further defines a general model and methodology for describing IPMCS applications in a form that is independent from a specific implementation. An application consists of one or more function block instances, interconnected by event connections and data connections as shown in figure 2. Process inputs and outputs define the interface of the application with the controlled industrial process.

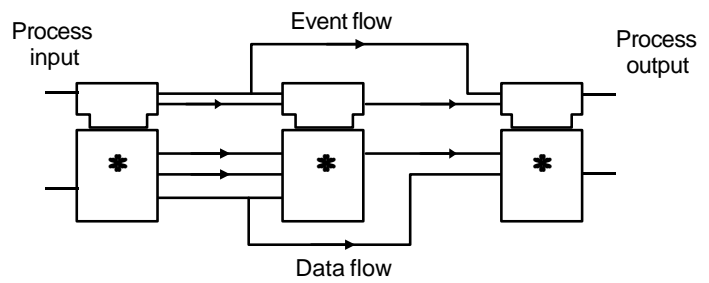

Figure 2. Part of a function block diagram of an IPMCS application.

We have defined the Industrial Process Terminator (IPT) construct, to represent, in the function block diagram, the sources and sinks of the application's events and data. Figure 3 , shows part of the metamodel we constructed using the Unified Modeling Language (UML), to clarify the function block related concepts [7]. The connection abstract-class represents either the connection between two

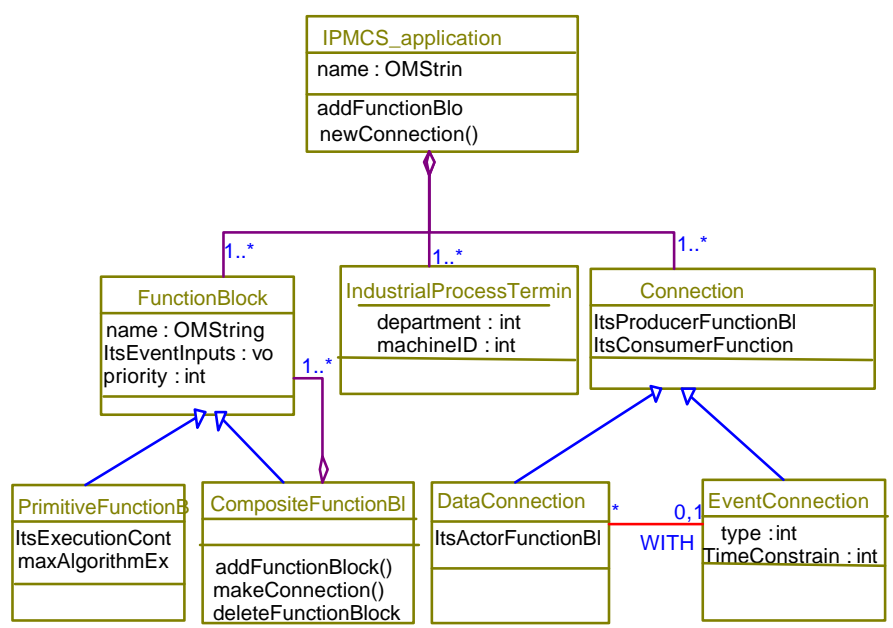

Figure 3. Part of the IPMCS application's metamodel.

function blocks or the one between a function block and an IPT. In any case, it is a data or an event connection. The aggregation of function blocks, connections and IPTs constitute the IPMCS application. The defined metamodel, constitutes a formal representation of the above concepts, and capture the key abstractions required for the design of an ESS.

\subsection{Distributed IPMCS applications}

The next phase of the development process, after the construction of the function block diagram, results in mapping the functionality captured by the IPMCS application into physical resources such as field devices. According to the IEC61499, devices may communicate with each other over one or more communication links, and may interface to controlled machines and processes. The IPMCS applications may be distributed among one or more devices, defining thus the general concept of the distributed IPMCS system that is shown in figure 4.

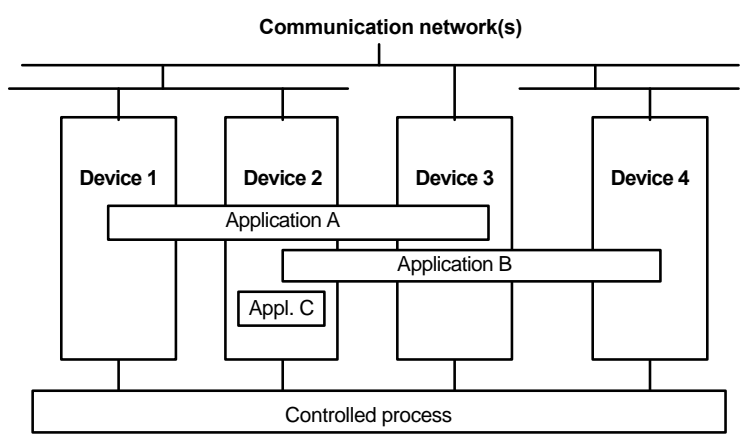

Figure 4. IPMCS applications may be distributed among field devices interconnected with one or more communication links

As far as, the field devices are interconnected with the same fieldbus, the distribution of the IPMCS application is a simple task. Data and event connections are mapped to the specific's fieldbus communication mechanisms and real time constraints are satisfied by the fieldbus nature. However, things are going to become more complicated, when the application's function blocks must be distributed among devices assigned to different fieldbuses of the same or different type.

This is a common situation in the industry, where a lot of proprietary fieldbuses have been installed in different time periods under different circumstances. If, for example, an enterprise wants to transfer data between two buildings, with each one having its own fieldbus, it has to interconnect the two heterogeneous fieldbuses. There is also a requirement, from the corporate level, to have integrated monitor and control of industrial processes assigned to different fieldbus segments, through the enterprise's intranet.

The obvious solution to the above problem, is to use interworking units to interconnect each fieldbus segment with the enterprise intranet. Although this solution, address the requirements for the integrated monitoring in corporate level, it does not satisfy the requirement for real-time interconnection between fieldbus segments. To satisfy this 
requirement, we were guided to adopt the network topology shown in figure 5. Our approach to use interworking units between each fieldbus and the enterprise intranet, leaves unchanged the already defined process of each fieldbus and only requires the extra effort for the interconnection of function blocks assigned to different fieldbus segments. This would result, in the least effort that the enterprise should spend over the configuration of the new system.

The communication subsystem must provide the quality of service required to meet the timing requirements. ATM is one of the successfully used technologies for the interconnection of fieldbuses [8][9]. However, to satisfy the key requirement of the simplicity of the communication system as well as the low cost of the equipment, switched Fast-Ethernet was selected.

\section{An open architecture for the interworking unit}

Interworking units, will theoretically appear to have intrinsic delays and performance limitations, but practically, they can be made to operate with very little disadvantage. Due to the strict timing constraints imposed by the application domain, the interworking unit is characterized as hard real-time. Data processing is expected to recognize and to react to events as soon as possible or even in the ideal case instantaneously. The key issue is how efficiently the interworking units can handle such requests and how the underlying communication subsystem can provide the required quality of service. A realtime OS was adopted for the implementation of the interworking unit, and a robust, modular and efficient architecture have been defined. For the design of an open interworking unit, we had to agree on some well-defined rules to govern the interactions among its subsystems. We use the term "architecture" to refer to the system's structure that consists of active modules, a mechanism to allow interaction among these modules and a set of rules that govern the interaction [10].

\subsection{Interworking unit's Active modules}

Figure 6, shows this part of the interworking unit's proposed architecture, that covers the operational phase of the system. It is composed of the following active modules:

\section{Local Fieldbus Proxy (LFP) \\ 2. Control Application (CA) \\ 3. Remote Fieldbus Proxies (RFP) \\ 4. OPC server}

The LFP module is used to abstract the specific fieldbus, to the IEC 61499 level so as the interoperability in fieldbus level may be achieved. It mainly consists of the local fieldbus exported function block proxies. A function block is considered as exported, if it provides or accepts an event or data from a function block located, either in the CA module or in a remote fieldbus. Another important task of the LFP module, is the coding and encoding of the proprietary fieldbus data representations to the IEC 61499 corresponding ones.

For the implementation of the LFP module, a wrapper layer is defined to adopt the proprietary fieldbus API. If we consider profibus as an example, the wrapper layer would

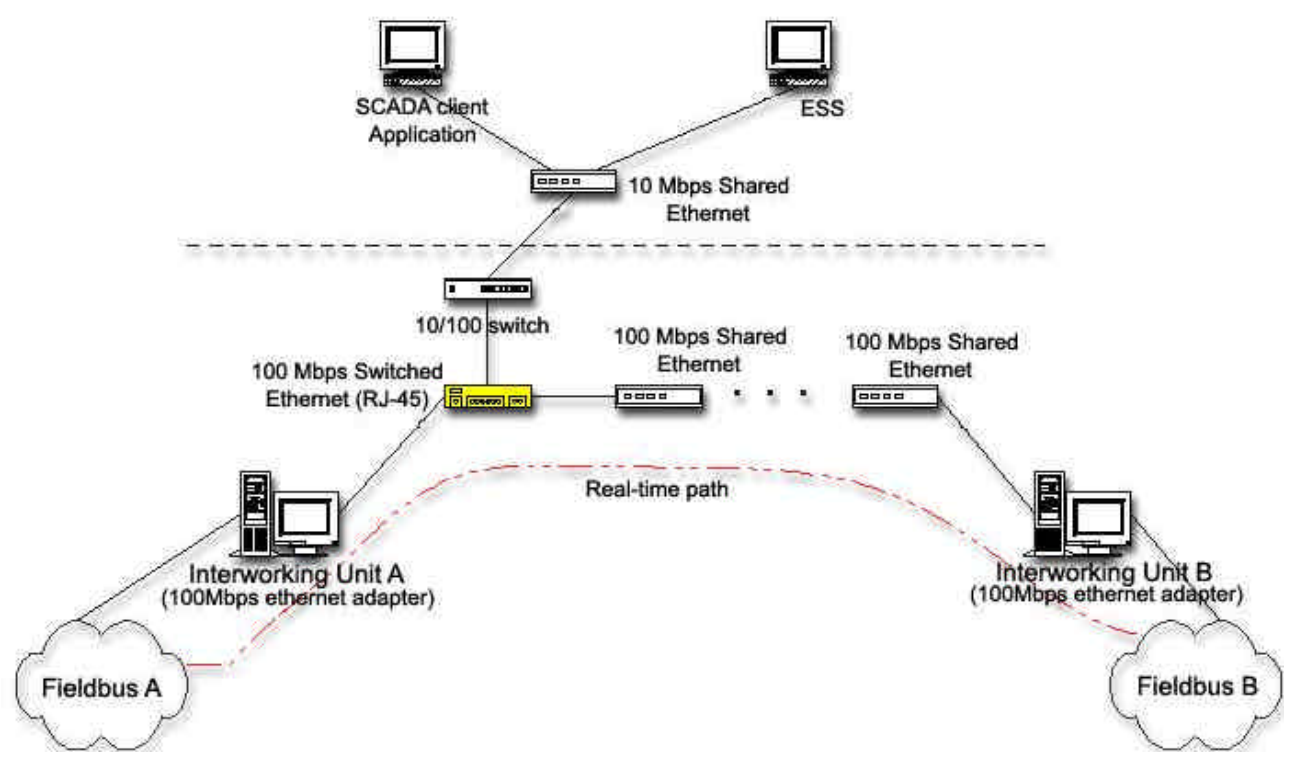

Figure 5. The proposed network topology for the real-time interconnection of fieldbus segments. 


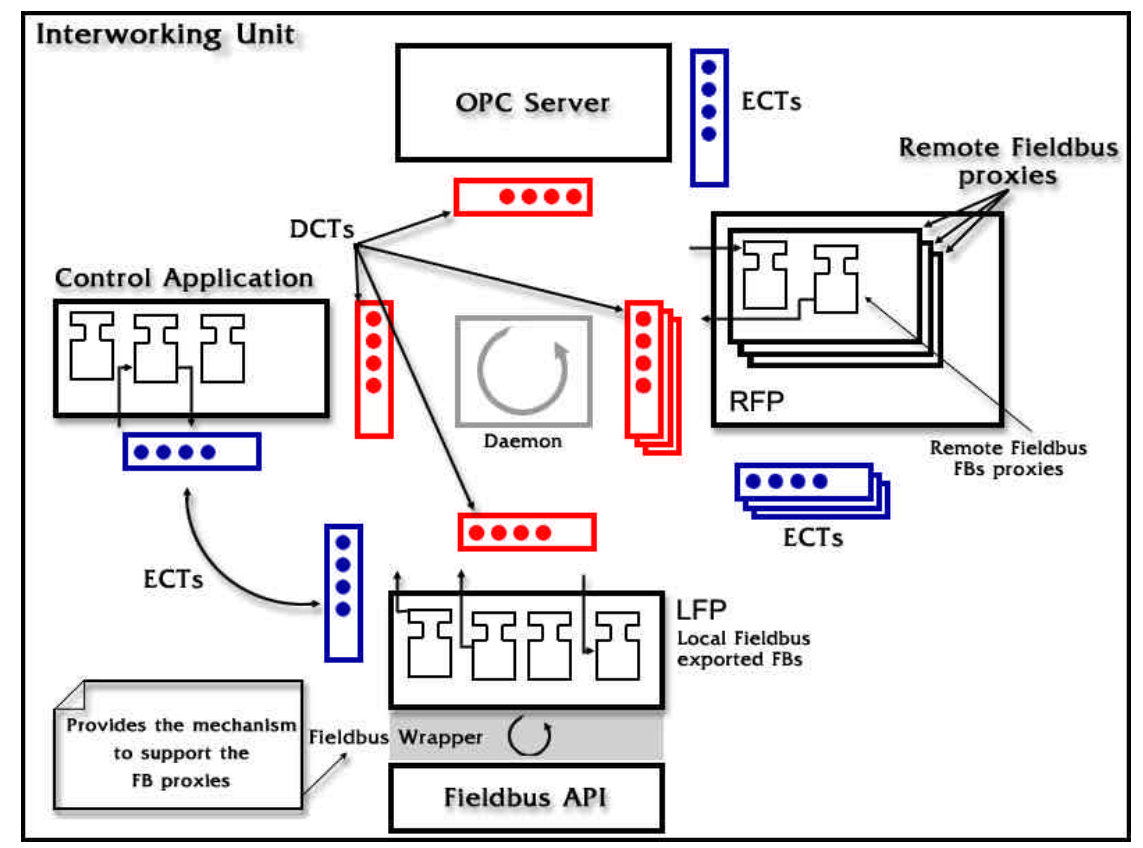

Figure 6. Architecture of the inteworking unit.

consists of a daemon process that has access to the Dual Port RAM of the profibus master card, to provide the underlying mechanism for the implementation of the function block proxies. In addition, the profibus wrapper must also implement the interconnections of function blocks located in different slaves.

The CA module, that is an optional module, is only required when there is a need to assign function blocks to the interworking unit. We usually discourage this design choice, but there are cases that such a decision is enforced by the system. In these cases, the performance of the interworking unit is degraded and an upper limit to the processing requirements of this module must be defined.

The RFP module acts as a container of all the remote fieldbus proxies that must be present in the interworking unit. For the interconnection of two fieldbuses we discriminate the following two cases:

1. A fieldbus proxy is defined in one of the interworking units. It is defined to contain both the producer and consumer function blocks of the remote fieldbus. In this case, there is only one remote fieldbus proxy through which the communication is obtained.

2. Two fieldbus proxies are defined, one per interworking unit. In this case, the remote fieldbus proxy contains only the producer function blocks.

The OPC server module is also an optional module that is required when there is a need for commercially available SCADA clients to be connected to the controlled process. This module acts as a wrapper to our architecture, to provide an OPC-compliant interface. We selected the OPC (OLE for Process Control) interface that seems to be the industry standard [11]. For the efficient development of the OPC server module, the following requirements should be met:

- It should support synchronous and asynchronous $\mathrm{read} / \mathrm{write}$ operations for data items

- It should serve the item requests, either through cache or through an immediate access to the device

- It should be able to queue requests and send the responses back in a single batch, keeping track of the requests

- It should guarantie that all relative items of an OPC group should be up to date

- It should be able, under several circumstances, to "push" data items to the client

- It should offer a safe shutdown procedure.

\subsection{Interaction Mechanism}

For the implementation of the mechanism to allow interaction among the above modules, we use a daemon process and a set of data structures, as is shown in figure 6.

There is an Event Connections Table (ECT) for each active module, except for the RFP module. In this case there is one ECT per each filedbus proxy contained in the RFP. The ECT contains, for every output event produced by the module, the information required by the daemon module to respond to this event. 
When an active module produces an event, it must send a request to the daemon module to handle this event. The daemon uses the information of the related ECT entry and forwards the event with the required data to the destination module. It is clear that there is a close relation to the WITH keyword of the function block notation. Actually the information captured by the WITH statements would be used by the ESS to properly initialize the ECTs.

The other data structure used is the Data Connection Table (DCT). DCTs contain the required information that must accompany each data connection of the module's function blocks that are considered as outputs and have as destinations, functions blocks assigned to other modules or fieldbuses. Such information includes the value, the time stamp, the status, etc. This information has of course to be in the IEC 61499 format.

\subsection{Rules of interaction}

The following rules have been defined in our attempt to meet all functional and non-functional (performance, extensibility and fault-tolerance) requirements:

1. When an active module produces data that are required by other modules, it properly updates the corresponding entries in its DCT.

2. When an event, that concerns another module, is produced inside an active module, the producer module notifies the daemon for the event occurrence by issuing a service request.

3. When the daemon is notified for the occurrence of an event, it access the information provided by the related entry of the producer module's ECT. Using this information, the daemon collects the required data items from DCTs and builds a packet, which pushes to the destination module.

4. Each service request is assigned a priority number.

5. An active module may issue an event that has another module as a destination.

6. An active module may issue an event service request to itself, to request from the daemon predefined data items.

7. ECTs may be updated during the operation phase, allowing for a dynamic reconfiguration of the application.

\section{The proposed 4-layer Architecture}

To proceed with the design and development of an ESS that is required to support the design, implementation, commissioning and operation of IPMCSs we have defined the 4-layer Architecture presented in Figure 7.

The bottom layer, that is the real world layer, is the actual IPMCS system. It consists of the controlled industrial processes, the fieldbus segments used to interconnect the field devices, the interworking units, the backbone, the enterprise intranet, the control room and the engineering workspace. The development of an efficient, robust and reliable such system is the target of any ESS. To this direction, the higher layers of abstraction must be directly assigned to this layer and this assignment must be invisible to the engineer. The required assignment mechanisms must be introduced and implemented by the ESS in such a way that the encapsulation of all the details of such assignments is of primary concern.

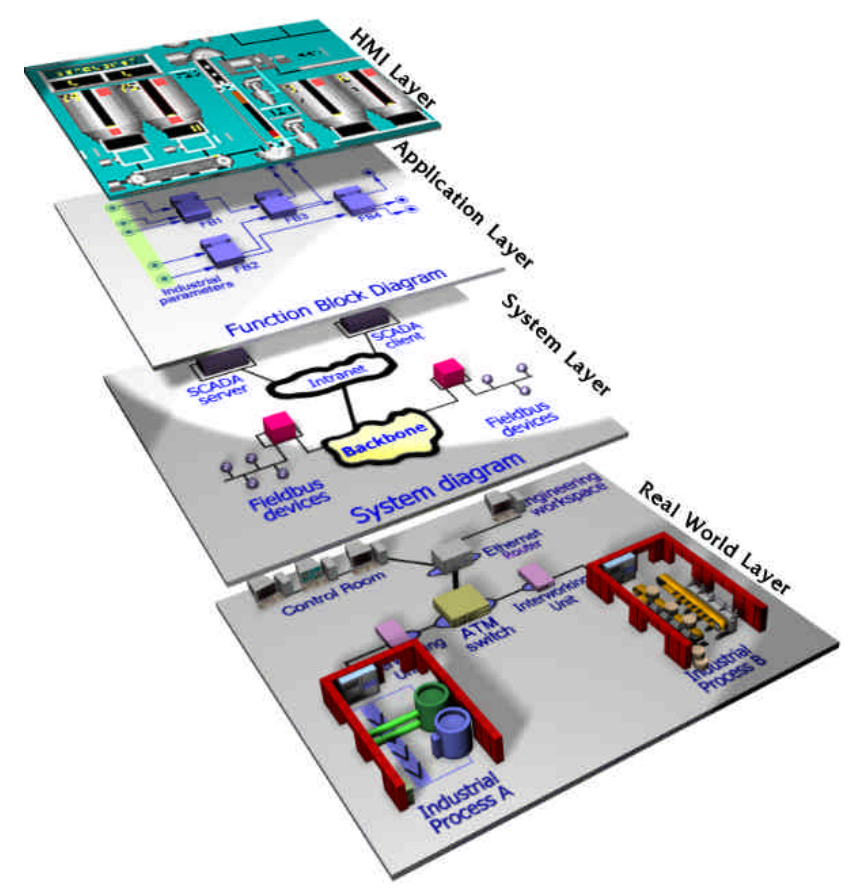

Figure 7. The 4-layer Architecture.

The next layer, that is the system layer, must include the required abstractions, used by the ESS to support the function block's distribution and assignment process. It consists of appropriate abstract representations of field devices, fieldbuses, interworking units, the backbone communication subsystem and the related interconnections. These abstract representations are in the form of proxies of the actual real world objects in the developer's workspace. The appropriate system level editor allows the engineer to construct the system design and directly map it to the lower level. One of the means used to support such a direct mapping is the device specification. This specification, that must be compliant with a common device model [12], accompanies the device in a user readable representation such as the one accomplished using XML [13]. The engineer, working in the real world layer, will connect the device in the fieldbus. He will then insert the device's representation into the system layer and, using the XML field device specification and the appropriate ESS's functionality, will 
build the machine-readable device model. This is the last step for the assignment process of the device's proxy, to the actual field device. An analogous process must be followed for every one of the other constructs of the system level diagram.

The third layer, that is called application layer, is used to represent the required constructs used in the design and implementation of the IPMCS applications. Function blocks, data and event connections, IPTs, and industrial process parameters, are the key abstractions and correspondingly the main building blocks of the application layer diagrams that are constructed by means of an application layer editor. The IPT instances of the diagram, must directly be mapped into the actual devices that interface the IPMCS with the controlled processes of the industrial environment. ESS must provide all the functionality required by the function block's distribution and assignment process to the system layer building blocks and mainly to field devices. Actions of the ESS that have no meaning in the application design phase, but refer to the configuration of the underlying communication system must be properly hided by encryption and encapsulation mechanisms.

Finally the HMI-layer captures all these abstractions that concern the development and operation of SCADA client systems. This layer seems to be partially in the scope of the ESS and has to be examined.

\section{Conclusions}

For the development of distributed IPMCS applications, we adopted the function block concept, to improve productivity in terms of re-use, reliability, flexibility and interoperability. We defined the architecture of an interworknig unit to address the problem of interconnecting heterogeneous fieldbus segments. Our architecture promotes re-usability, obtains interoperability in the fieldbus level and allows a uniform development of applications from the ESS point of view. It also considers the interface to the commercially available SCADA client systems that are OPC-compliant. We constructed the whole architecture having in mind issues such as modularity, expandability, robustness, and flexibility. We have found our architecture very helpful in the development process of our function block oriented Engineering Support System. It helped us to easily identify services for application management, such as function block creation, deletion, interconnection and activation. The importance of such services is expected to increase along with the demand for system agility.

We have designated four layers of abstraction and constructed a 4-layer architecture of the IPMCSs. We have found this architecture very helpful in the process of capturing the key abstractions, that will become the basis for the development of the function block oriented ESS. The architecture has defined the main directions that an ESS must address and is playing an important role during the development of our ESS.

We hope this work will help in the direction of developing interoperable IEC 61499-compliant systems that will provide the ability to the industry to utilize heterogeneous software environments, with IEC 61499-compliant software tools and devices available from a wide selection of vendors.

\section{Acknowledgements}

This work has been funded in part by the Greek General Secretariat for Research and Technology in the context of the PENED 99 ED 469 project. We gratefully thank Chris Coulamas and Loukas Mandalos members of the ARTIO development team, for their helpful discussions. Loukas Mandalos suggested the use of switched Fast Ethernet instead of ATM.

\section{References}

[1] IEC Technical Committee TC65/WG6, "IEC61499 Industrial-Process Measurement and Control Specification", IEC Draft 2000

[2] IEC SUB COMMITTEE No. 65C: DIGITAL COMMUNICATIONS, WORKING GROUP 7: FUNCTION BLOCKS for PROCESS CONTROL , "IEC1804 General Requirements", IEC Draft 1999

[3] Softing's Profigate Datasheet, http://www.softing.com

[4] SIMATIC OPC Server, User guide http://www.siemens.com

[5] SST-X-Link gateway, http://www.mysst.com

[6] R.W. Lewis, "Modeling Distributed Control Systems Using IEC61499 Function Blocks", Technical Articles, URL: http://www.searcheng.co.uk/ selection/ control/tech.htm

[7] "OMG Unified Modeling Language Specification", Version 1.3, First Edition, Object Management Group Inc. March 2000.

[8] O. Kunert, “ Interconnecting fieldbuses through ATM", IEEE international workshop on factory communication systems, 1997.

[9] C.Cseh, M.Jansen, J.Jasperneite, "ATM networks for factory communication", $7^{\text {th }}$ IEEE International Conference on Emerging Technologies and Factory Automation, 1999.

[10] Maarten Boasson, "The Artistry of Software Architecture", IEEE Software, November 1995, vol. 12 No 6.

[11] OPC Task Force, OLE for Process Control: Overview, OPC Foundation, Oct. 1998

[12] K. Thramboulidis, A. Prayati, "Field Device Specification for the Development of Function Block Oriented ESSs", submitted to ETFA 2001.

[13] Extensible Markup Language (XML) 1.0 (Second Edition), W3C Recommendation, 6 October 2000. 